\title{
Studying $\Lambda^{*}$ resonances in the $p \bar{p} \rightarrow \Lambda \bar{\Lambda} \eta$ reaction
}

\author{
Bo-Chao Liu ${ }^{*}$ and Ke Wang \\ School of Physics, Xi'an Jiaotong University, Xi'an, Shannxi 710049, China
}

(Received 7 October 2019; accepted 4 June 2020; published 26 June 2020)

\begin{abstract}
In this work, we make a theoretical study on the $p \bar{p} \rightarrow \Lambda \bar{\Lambda} \eta$ reaction for antiproton beam energy from threshold to $4 \mathrm{GeV}$ within an effective Lagrangian approach and isobar model. By assuming this reaction is dominated by the excitation of $\Lambda$ and $\bar{\Lambda}$ resonances in intermediate states, we calculate the total cross sections and give the predictions of the angular distribution and invariant mass spectrum of final particles. In particular, we discuss the possibility to verify the existence of a narrow $\Lambda$ resonance found in the process of $K^{-} p \rightarrow \eta \Lambda$ in the present reaction. It shows that the $p \bar{p} \rightarrow \bar{\Lambda} \Lambda \eta$ reaction can provide us with valuable information about the $\Lambda$ resonances having significant couplings to $\bar{K} N$ and $\Lambda \eta$ channels. Thus the experimental data of this reaction will be a good supplement to the $\bar{K} N \rightarrow \eta \Lambda$ scattering data for studying $\Lambda$ resonances.
\end{abstract}

DOI: 10.1103/PhysRevD.101.114030

\section{INTRODUCTION}

The study on the properties of $\Lambda$ resonances constitutes one important part of the research in the baryon spectroscopy, which offers us useful information about the strong interaction in the nonperturbative energy region and also tests of our knowledge in the strange particle channels. Up to now, most of the knowledge about $\Lambda$ resonances is from the analysis of the data in the $\bar{K} N$ and $\pi \Sigma$ channels. Studies on other channels, although very important, are still relatively lacking. Due to isospin conservation, the $\eta \Lambda$ channel is of special interest because it only couples to $\Lambda$ resonance, which offers a relatively clean channel for studying the properties of the $\Lambda$ resonances. But even with this advantage, the status of current knowledge on the coupling of $\Lambda$ resonances to $\eta \Lambda$ channel is still not satisfying. In the Particle Data Group (PDG) book [1], there is only one $\Lambda^{*}$ state, i.e., $\Lambda(1670)$, has well-established coupling with $\eta \Lambda$ channel. The decay branch ratio of other $\Lambda$ resonances to this channel is still not well identified. It is possible that other resonances indeed have weak coupling with this channel and are therefore hard to study their couplings with $\eta \Lambda$. However, the relatively poor quality of experimental data in this channel is also a potentially important reason.

The Crystal Ball Collaboration data on the reaction $K^{-} p \rightarrow \eta \Lambda$ near threshold published in 2012 have much

\footnotetext{
*liubc@xjtu.edu.cn
}

Published by the American Physical Society under the terms of the Creative Commons Attribution 4.0 International license. Further distribution of this work must maintain attribution to the author(s) and the published article's title, journal citation, and DOI. Funded by SCOAP . higher accuracy than before, which offers a good basis to investigate the reaction mechanism of this reaction and to extract the properties of $\Lambda$ resonances in the $\eta \Lambda$ channel. Based on the new data an analysis within an effective Lagrangian approach and isobar model was performed in Refs. [2,3]. The main findings are, although the $\Lambda(1670)$ gives the dominant contribution near threshold, the bowl structure appearing in the angular distribution may indicate a new narrow resonance. It was shown that the experimental data supported the existence of a $D_{03}$ resonance with $\mathrm{M}=1668.5 \pm 0.5 \mathrm{MeV}$ and $\Gamma=1.5 \pm 0.5 \mathrm{MeV}$ (denoted as $\Lambda_{D}^{*}$ for convenience). Due to the very narrow width, this $\Lambda$ resonance is obviously not any existing $\Lambda$ resonance in the PDG book. The possible existence of a narrow $\Lambda$ resonance in this channel was confirmed by another group based on a coupled-channel analysis [4,5]. However, in their analysis the proposed narrow resonance has the quantum numbers $J^{P}=\frac{3}{2}{ }^{+}$(hereafter referred to as $\Lambda_{P}^{*}$ ). Very interestingly, a narrow enhancement lying near the $\eta \Lambda$ threshold was also found in the mass spectrum of $K^{-} p$ in the decay of $\Lambda_{c} \rightarrow p K^{-} \pi^{+}$at Belle [6]. Until now, the origin of this enhancement is still not well identified. Very recently, it was argued that the enhancement might be caused by kinematical singularity [7]. However, as stated by the authors of this work, partial wave analysis is still needed to distinguish various scenarios. Obviously, to establish whether the narrow resonance exists or not, further studies on both theoretical and experimental sides are still needed.

The PANDA experiment [8] at the Facility for Antiproton and Ion Research (FAIR) will be carried out in the near future, which is well suited for exploring the spectroscopy of strange and charmed baryons. Such experiment will definitely offer valuable data for improving our 
knowledge of the strong interaction and of hadron spectroscopy. Encouraged by the prospect, there have been a series of theoretical investigations on the new opportunities for studying the baryon spectroscopy in $N \bar{N}$ collisions [9-14]. In their studies, they mainly focused on the production of charmed hadrons. In this work, we attempt to show that the reaction $p \bar{p} \rightarrow \Lambda \bar{\Lambda} \eta$ may be a suitable place to explore the properties of $\Lambda$ resonances. To our best knowledge, there is still no experimental data available for this reaction. Our calculation will mainly be based on an effective Lagrangian approach and isobar model. In our model, the $\Lambda$ resonances are excited due to the $K$ and $K^{*}$ meson exchanges between the initial proton and antiproton. Thus this reaction offers the possibility to explore the $\Lambda$ resonances having significant coupling with $\bar{K} N\left(\bar{K}^{*} N\right)$ and $\Lambda \eta$ channels. Until now, only the $\Lambda(1670)$ is known to have significant coupling to these channels. If the narrow resonance mentioned above indeed exists, it should also play a role in this reaction. In addition, the present reaction may also proceed through the excitation of mesonic resonances in the intermediate states, which finally decay to $\Lambda \bar{\Lambda}$. In our model, the production of such states is induced by the exchange of nucleon or nucleon resonance [e.g., $N(1535)]$ between initial proton and antiproton. For nucleon exchange, its contribution should be suppressed due to the vanishing $N N \eta$ coupling [15]. While for nucleon resonance exchange, its contribution can not be well estimated due to the poor knowledge of the $\bar{N} N^{*} \rightarrow \Lambda \bar{\Lambda}$ process. Thus we choose to ignore these contributions. ${ }^{1}$ In this work, we shall consider the contributions from the $\Lambda(1670)$ and the possible narrow $\Lambda$ resonance with considering both the $P_{03}$ and $D_{03}$ assignments for its quantum numbers. Most of the model parameters are determined by fitting the data of the $K^{-} p \rightarrow \eta \Lambda$ reaction. The predictions of the angular distribution, invariant mass spectrum and Dalitz plot are presented, which should be useful for future comparisons with data and looking for the possible narrow $\Lambda$ resonance.

This paper is organized as follows. In Sec. II, the theoretical framework and amplitudes are presented for the reaction $p \bar{p} \rightarrow \bar{\Lambda} \Lambda \eta$. In Sec. III, the numerical results are presented with some discussions. Finally, the paper ends with a short summary in Sec. IV.

\section{THEORETICAL FORMALISM}

In this work, we investigate the $p \bar{p} \rightarrow \Lambda \bar{\Lambda} \eta$ reaction within an effective Lagrangian approach and isobar model. We assume that this reaction is dominated by the excitation

\footnotetext{
${ }^{1}$ For the purpose of studying the $\Lambda$ resonances, the uncertainties due to these contributions can be controlled experimentally. When some resonance decaying to the $\Lambda \bar{\Lambda}$ channel contributes significantly and its contribution overlap with the narrow $\Lambda$ resonance, it is possible to separate their contributions or their bands in the Dalitz plot by choosing a different beam energy.
}

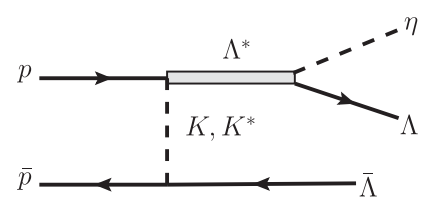

(a)

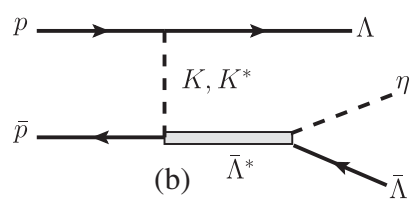

(b)
FIG. 1. Model for the reaction $p \bar{p} \rightarrow \Lambda \bar{\Lambda} \eta$.

of $\Lambda$ and $\bar{\Lambda}$ resonances in the intermediate states with considering the contributions from the $\Lambda(1670)$ and a very narrow $\Lambda_{D}^{*} / \Lambda_{P}^{*}$ resonance suggested in Refs. [2-5]. The basic Feynman diagrams are depicted in Fig. 1.

The effective Lagrangians describing the $K N \Lambda$ and $K^{*} N \Lambda$ interactions can be given as

$$
\begin{gathered}
\mathcal{L}_{K N \Lambda}=-i g_{K N \Lambda} \bar{\Psi}_{N} \gamma_{5} \Psi_{\Lambda} \Phi_{K}+\text { H.c. } \\
\mathcal{L}_{K^{*} N \Lambda}=g_{K^{*} N \Lambda} \bar{\Psi}_{N}\left(\gamma^{\mu} K_{\mu}^{*}-\frac{\kappa_{K^{*}}}{2 m_{N}} \sigma^{\mu \nu} \partial_{\nu} K_{\mu}^{*}\right) \Psi_{\Lambda}+\text { H.c. }
\end{gathered}
$$

The value of $g_{N K \Lambda}$ can be determined by the SU(3) predictions, and we adopt $g_{N K \Lambda}=-13.24$ in our calculations $[16,17]$. For the coupling constants $g_{K^{*} N \Lambda}$ and $\kappa_{K^{*}}$, we take their values from the Nijmegen potential, i.e., $g_{K^{*} N \Lambda}=4.26$ and $\kappa_{K^{*}}=2.66[18,19]$.

The relevant interaction Lagrangians involving the $\Lambda(1670)$ or the $\Lambda_{D}^{*} / \Lambda_{P}^{*}$ resonances are used in the same forms as in Refs. [2,3],

$$
\begin{gathered}
\mathcal{L}_{\Lambda(1670) \Lambda \eta}=g_{\Lambda(1670) \Lambda \eta} \bar{\Lambda} \Lambda^{*} \eta+\text { H.c. } \\
\mathcal{L}_{\Lambda(1670) K N}=g_{\Lambda(1670) K N} \bar{\Lambda}^{*} \bar{K} N+\text { H.c. } \\
\mathcal{L}_{\Lambda(1670) K^{*} N}=i g_{\Lambda(1670) K^{*} N} \bar{\Lambda}^{*} \gamma_{5} \gamma^{\mu} K_{\mu}^{*} N+\text { H.c. } \\
\mathcal{L}_{\Lambda_{D}^{*} K N}=\frac{f_{\Lambda_{D}^{*} K N}}{m_{K}} \partial_{\mu} \bar{K} \bar{\Lambda}^{* \mu} \gamma_{5} N+\text { H.c. } \\
\mathcal{L}_{\Lambda_{D}^{*} \Lambda \eta}=\frac{f_{\Lambda_{D}^{*} \Lambda \eta}}{m_{\eta}} \partial_{\mu} \eta \bar{\Lambda}^{* \mu} \gamma_{5} \Lambda+\text { H.c. } \\
\mathcal{L}_{\Lambda_{P}^{*} K N}=\frac{f_{\Lambda_{P}^{*} K N}}{m_{K}} \partial_{\mu} \bar{K} \bar{\Lambda}^{* \mu} N+\text { H.c. } \\
\mathcal{L}_{\Lambda_{P}^{*} \Lambda \eta}=\frac{f_{\Lambda_{P}^{*} \Lambda \eta}}{m_{\eta}} \partial_{\mu} \eta \bar{\Lambda}^{* \mu} \Lambda+\text { H.c. }
\end{gathered}
$$

The coupling constant $g_{\Lambda(1670) K^{*} N}=0.753$ is taken from Ref. [20], where its value is obtained based on a chiral quark model. For the $\Lambda_{D}^{*} \bar{K} N$ and $\Lambda_{D}^{*} \eta \Lambda$ couplings, we follow the results in Refs. [2,3], where the relevant coupling constants are fitted to the experimental data of the $K^{-} p \rightarrow \eta \Lambda$ reaction (Scenario I). The obtained parameters are shown in Table I. To get the parameters for the $P_{03}$ assignment, we fit them to the same data set as in the Scenario I but assuming the new $\Lambda$ resonance is a $P_{03}$ state (Scenario II). The obtained mass and 
TABLE I. Parameters obtained by fitting to the total and differential cross sections of the $K^{-} p \rightarrow \eta \Lambda$ reaction.

\begin{tabular}{|c|c|c|c|c|c|c|}
\hline Scenario & Considered resonance & Product of coupling constants & Relative phase $\left(\phi_{\alpha}\right)$ & Mass $(\mathrm{MeV})$ & Width $(\mathrm{MeV})$ & $\chi^{2} /$ d.o.f. \\
\hline \multirow[t]{2}{*}{ I } & $\Lambda(1670)$ & $g_{\Lambda(1670) \Lambda \eta} g_{\Lambda(1670) \bar{K} N}=0.30 \pm 0.03$ & 0 & $1672.5 \pm 1.0$ & $24.5 \pm 2.7$ & 0.88 \\
\hline & $\Lambda_{D}^{*}$ & $f_{\Lambda_{D}^{*} \bar{K} N} f_{\Lambda_{D}^{*} \Lambda \eta}=28.2 \pm 2.4$ & $5.66 \pm 0.47$ & $1668.5 \pm 0.5$ & $1.5 \pm 0.5$ & \\
\hline \multirow[t]{2}{*}{ II } & $\Lambda(1670)$ & $g_{\Lambda(1670) \Lambda \eta} g_{\Lambda(1670) \bar{K} N}=0.32 \pm 0.03$ & 0 & $1672.2 \pm 0.8$ & $27.6 \pm 1.0$ & 0.86 \\
\hline & $\Lambda_{P}^{*}$ & $f_{\Lambda_{P}^{*} \bar{K} N} f_{\Lambda_{P}^{*} \Lambda \eta}=2.98 \pm 0.08$ & $0.61 \pm 0.08$ & $1663.6 \pm 0.5$ & $11.0 \pm 1.4$ & \\
\hline III & $\Lambda(1670)$ & $g_{\Lambda(1670) \Lambda \eta} g_{\Lambda(1670) \bar{K} N}=0.28 \pm 0.02$ & - & $1671.5 \pm 0.2$ & $23.2 \pm 0.2$ & 1.22 \\
\hline
\end{tabular}

width of the narrow resonance are consistent with the results in Ref. [5] within uncertainties. Note that we also calculate the predictions of the $\Lambda$ polarization for the $K^{-} p \rightarrow \eta \Lambda$ reaction and find that in Scenario II the predictions seem incompatible to the available data (see also Fig. 20 of Ref. [4]). So more accurate $\Lambda$ polarization data of the $K^{-} p \rightarrow \eta \Lambda$ reaction will be helpful to clarify the quantum numbers of this narrow resonance.

Because hadrons cannot be treated as elementary particles in the energy region under study, it is necessary to take into account the internal structures and off-shell effects. In phenomenological models, this is usually done by introducing form factors. In this work, we adopt the following form factor for various meson exchange vertices,

$$
F_{M}(q)=\frac{\Lambda_{M}^{2}-m^{2}}{\Lambda_{M}^{2}-q^{2}}
$$

where $\Lambda_{M}, m$, and $q$ are the cutoff parameter, the mass of the exchanged particle, and the exchanged momentum. The cutoff parameters for the $K N \Lambda$ and $K^{*} N \Lambda$ vertices are adopted as $\Lambda_{K}=1.1 \mathrm{GeV}$ and $\Lambda_{K^{*}}=0.9 \mathrm{GeV}[16,17]$, respectively. While, the cutoff parameters for the $\Lambda^{*} \bar{K} N$ vertices are not well determined in literatures. In present work, we use the same value as that for the $\Lambda \bar{K} N$ vertex and the uncertainties due to this parameter will be discussed in Sec. III.

The propagators for the $\Lambda(1670), \Lambda_{P / D}^{*}, K$, and $K^{*}$ are adopted as the following forms:

$$
\begin{gathered}
G_{\Lambda(1670)}^{ \pm}(q)=\frac{i\left(\not q \pm M_{\Lambda(1670)}\right)}{q^{2}-M_{\Lambda(1670)}^{2}+i M_{\Lambda(1670)} \Gamma_{\Lambda(1670)}}, \\
G_{\Lambda_{P / D}^{*}}^{ \pm}(q)=\frac{i\left(\not q \pm M_{\Lambda_{P / D}^{*}}\right)}{q^{2}-M_{\Lambda_{P / D}^{*}}^{2}+i M_{\Lambda_{P / D}^{*}} \Gamma_{\Lambda_{P / D}^{*}}}\left[-g_{\mu \nu}+\frac{1}{3} \gamma_{\mu} \gamma_{\nu}\right. \\
\left. \pm \frac{1}{3 M_{\Lambda_{P / D}^{*}}}\left(\gamma_{\mu} q_{\nu}-\gamma_{\nu} q_{\mu}\right)+\frac{2}{3 M_{\Lambda_{P / D}^{*}}^{2}} q_{\mu} q_{\nu}\right] \\
G_{K}(q)=\frac{i}{q^{2}-m_{K}^{2}}, \\
G_{K^{*}}^{\mu \nu}(q)=i \frac{-g^{\mu \nu}+q^{\mu} q^{\nu} / m_{K^{*}}^{2}}{q^{2}-m_{K^{*}}^{2}}
\end{gathered}
$$

where the superscript + and - correspond to particle and antiparticle, respectively.

With the ingredients given above, the amplitudes for various diagrams can be written by following the Feynman rules. Here we present the individual amplitudes explicitly,

$$
\begin{aligned}
& \mathcal{M}_{a, K}^{\Lambda(1670)}=g_{\Lambda(1670) \Lambda \eta} g_{N K \Lambda} g_{\Lambda^{*} \bar{K} N} \bar{u}_{\Lambda, s_{\Lambda}} G_{\Lambda^{*}}^{(+)}(P) F_{K}(q) \\
& \times u_{p, s_{p}} G_{K}(q) \bar{v}_{\bar{p}, s_{\bar{p}}} \gamma_{5} v_{\bar{\Lambda}, s_{\bar{\Lambda}}} \\
& \mathcal{M}_{b, K}^{\Lambda(1670)}=-g_{\Lambda(1670) \Lambda \eta} g_{N K \Lambda} g_{\Lambda^{*} \bar{K} N} \bar{v}_{\bar{p}, s_{\bar{p}}} G_{\Lambda^{*}}^{(-)}\left(P^{\prime}\right) F_{K}\left(q^{\prime}\right) \\
& \times v_{\bar{\Lambda}, s_{\bar{\Lambda}}} G_{K}\left(q^{\prime}\right) \bar{u}_{\Lambda, s_{\Lambda}} \gamma_{5} u_{p, s_{p}} \\
& \mathcal{M}_{a, K^{*}}^{\Lambda(1670)}=-g_{\Lambda(1670) \Lambda \eta} g_{K^{*} N \Lambda} g_{\Lambda^{*} K^{*} N} \bar{u}_{\Lambda, s_{\Lambda}} G_{\Lambda^{*}}^{(+)}(P) \gamma_{5} \gamma_{\mu} F_{K^{*}}(q) \\
& \times u_{p, s_{p}} G_{K^{*}}^{\mu \nu}(q) \bar{v}_{\bar{p}, s_{\bar{p}}}\left(\gamma_{\nu}-i \frac{\kappa_{K^{*}}}{2 m_{N}} \sigma_{\nu \rho} q^{\rho}\right) v_{\bar{\Lambda}, s_{\bar{\Lambda}}} \\
& \mathcal{M}_{b, K^{*}}^{\Lambda(1670)}=g_{\Lambda(1670) \Lambda \eta} g_{K^{*} N \Lambda} g_{\Lambda^{*} K^{*} N} \bar{v}_{\bar{p}, s_{\bar{p}}} \gamma_{5} \gamma_{\mu} F_{K^{*}}\left(q^{\prime}\right) G_{\Lambda^{*}}^{(-)}\left(P^{\prime}\right) \\
& \times v_{\bar{\Lambda}, s_{\bar{\Lambda}}} G_{K^{*}}^{\mu \nu}\left(q^{\prime}\right) \bar{u}_{\Lambda, s_{\Lambda}}\left(\gamma_{\nu}-i \frac{\kappa_{K^{*}}}{2 m_{N}} \sigma_{\nu \rho} q^{\prime \rho}\right) u_{p, s_{p}} \\
& \mathcal{M}_{a}^{\Lambda_{D}^{*}}=e^{i \phi_{\alpha}} \frac{g_{N K \Lambda} f_{\Lambda_{D}^{*} \bar{K} N} f_{\Lambda_{D}^{*} \Lambda \eta}}{m_{\eta} m_{K}} \bar{u}_{\Lambda, s_{\Lambda}} \gamma_{5} G_{\Lambda^{*}}^{(+) \mu \nu}(P) p_{\mu}^{\eta} q_{\nu} \\
& \times F_{\Lambda^{*}}(q) \gamma_{5} u_{p, s_{p}} G_{K}(q) \bar{v}_{\bar{p}, s_{\bar{p}}} \gamma_{5} v_{\bar{\Lambda}, s_{\bar{\Lambda}}} \\
& \mathcal{M}_{b}^{\Lambda_{D}^{*}}=-e^{i \phi_{\alpha}} \frac{g_{N K \Lambda} f_{\Lambda_{D}^{*} \bar{K} N} f_{\Lambda_{D}^{*} \Lambda \eta}}{m_{\eta} m_{K}} \bar{v}_{\bar{p}, s_{\bar{p}}} \gamma_{5} G_{\Lambda^{*}}^{(-) \mu \nu}\left(P^{\prime}\right) p_{\nu}^{\eta} \\
& \times q_{\mu}^{\prime} F_{\Lambda^{*}}\left(q^{\prime}\right) \gamma_{5} v_{\bar{\Lambda}, s_{\bar{\Lambda}}} G_{K}\left(q^{\prime}\right) \bar{u}_{\Lambda, s_{\Lambda}} \gamma_{5} u_{p, s_{p}} \\
& \mathcal{M}_{a}^{\Lambda_{P}^{*}}=e^{i \phi_{\alpha}} \frac{g_{N K \Lambda} f_{\Lambda_{P}^{*} \bar{K} N} f_{\Lambda_{P}^{*} \Lambda \eta}}{m_{\eta} m_{K}} \bar{u}_{\Lambda, s_{\Lambda}} G_{\Lambda^{*}}^{(+) \mu \nu}(P) p_{\mu}^{\eta} q_{\nu} \\
& \times F_{\Lambda^{*}}(q) u_{p, s_{p}} G_{K}(q) \bar{v}_{\bar{p}, s_{\bar{p}}} \gamma_{5} v_{\bar{\Lambda}, s_{\bar{\Lambda}}} \\
& \mathcal{M}_{b}^{\Lambda_{P}^{*}}=-e^{i \phi_{\alpha}} \frac{g_{N K \Lambda} f_{\Lambda_{P}^{*} \bar{K} N} f_{\Lambda_{P}^{*} \Lambda \eta}}{m_{\eta} m_{K}} \bar{v}_{\bar{p}, s_{\bar{p}}} G_{\Lambda^{*}}^{(-) \mu \nu}\left(P^{\prime}\right) p_{\nu}^{\eta} q_{\mu}^{\prime} \\
& \times F_{\Lambda^{*}}\left(q^{\prime}\right) v_{\bar{\Lambda}, s_{\bar{\Lambda}}} G_{K}\left(q^{\prime}\right) \bar{u}_{\Lambda, s_{\Lambda}} \gamma_{5} u_{p, s_{p}}
\end{aligned}
$$

In the above formulas, the letters in the parentheses indicate the momentum of the exchanged particles and $p_{\eta}$ denotes the momentum of the $\eta$ in the final state.

Based on the individual scattering amplitudes presented above, the general differential cross section of $p \bar{p} \rightarrow \Lambda \bar{\Lambda} \eta$ reads 


$$
\begin{aligned}
d \sigma= & \frac{1}{16} \frac{1}{\sqrt{\left(p_{p} \cdot p_{\bar{p}}\right)^{2}-m_{p}^{4}}} \frac{1}{(2 \pi)^{5}} \sum_{s_{i}, s_{f}}\left|\mathcal{M}_{f i}\right|^{2} \\
& \cdot \prod_{a=1}^{3} \frac{d^{3} p_{a}}{2 E_{a}} \delta^{4}\left(P_{i}-P_{f}\right)
\end{aligned}
$$

where $\mathcal{M}_{f i}$ represents the total amplitude, $P_{i}$ and $P_{f}$ represent the sum of all the momenta in the initial and final states, respectively. $p_{a}$ denotes the momenta of the three particles in the final state.

Before presenting the calculated results, we need to discuss the possible effects of the $p \bar{p}$ initial state interaction (ISI) and $\Lambda \bar{\Lambda}$ final state interaction (FSI) in the present reaction. It is known that the ISI may have important effects on the meson production in nucleon-nucleon collisions $[21,22]$, where the ISI reduces the cross section by an over factor with slight energy dependence. The study on the $p \bar{p} \rightarrow \Lambda_{c} \bar{\Lambda}_{c}$ reaction also shows that the ISI effect may reduce the cross section by a factor of 100 [10]. So it is natural to expect that the ISI effect may also be important for the reaction under study. When we consider the energy region near threshold, the interaction between final $\Lambda$ and $\bar{\Lambda}$ may also become important [23]. A reliable description of the FSI between the $\Lambda$ and $\bar{\Lambda}$ will rely on a good understanding of the $\Lambda \bar{\Lambda}$ interaction, for which our knowledge is still rather limited due to the absence of data. Therefore an accurate description of the FSI between $\Lambda$ and $\bar{\Lambda}$ is still not possible. To take into account the ISI effect, we adopt a phenomenological approach as in Refs. [24,25]. Interestingly, in a recent work [11], the authors have adopted the same approach and applied it to study the $p \bar{p} \rightarrow \bar{\Lambda}_{c}^{-} \Lambda_{c}^{+}$reaction. In their work, the parameters for the ISI were checked by reproducing the near threshold cross sections predicted by Juelich model, in which model ISI is taken into account more rigorously. Using the same parameters, they can also successfully reproduce the cross sections of the $p \bar{p} \rightarrow \Lambda \bar{\Lambda}$ reaction near threshold without considering FSI effect explicitly. For simplicity, in this work we choose to follow the approach in Ref. [11] and adopt their parameters. Thus we assume the effect of FSI has been effectively absorbed into the model parameters. Here we want to note even though we treat the ISI and FSI in a model dependent way, the main conclusions of the present work should not be changed significantly since our primary goal is to have an order of magnitude estimation of the total cross sections and to investigate the relative importance of various $\Lambda$ resonances in this reaction.

\section{RESULTS AND DISCUSSIONS}

With the formulas and ingredients given in last section, the total and differential cross sections can be calculated in a straightforward way and we present the results in this section. To investigate the roles of the $\Lambda(1670)$ and the possible narrow resonance in the $p \bar{p} \rightarrow \Lambda \bar{\Lambda} \eta$ reaction, we will consider three scenarios. First, we include the contributions from both the $\Lambda(1670)$ and the narrow $\Lambda_{D}^{*}$ in the reaction (Scenario I). Second, we adopt the assumption that the narrow resonance is a $P_{03}$ state as in Refs. [4,5] and consider its contribution in this reaction (Scenario II). Finally, we consider the case that the narrow resonance does not exist and thus there is no contribution from the narrow resonance (Scenario III). For all the three scenarios, the parameters of the models such as the coupling constants and relative phases are determined by fitting the total and differential cross sections of the $K^{-} p \rightarrow \eta \Lambda$ reaction, where these resonances play important roles. The adopted parameters have been listed in Table I.

In Fig. 2(a), we show the total cross sections and the individual contributions of various resonances in Scenarios I and II. As shown in Fig. 2(a), the $\Lambda_{D}^{*}$ contribution is suppressed at the very near threshold region and the $\Lambda(1670)$ gives the most important contribution at first. When the center of mass energy approaches the threshold of $\Lambda_{D}^{*} \bar{\Lambda} / \bar{\Lambda}_{D}^{*} \Lambda\left(\sqrt{s}=M_{\Lambda_{D}^{*}}+M_{\bar{\Lambda}}\right)$, the production of $\Lambda_{D}^{*}$ starts to play more important role and causes the strong
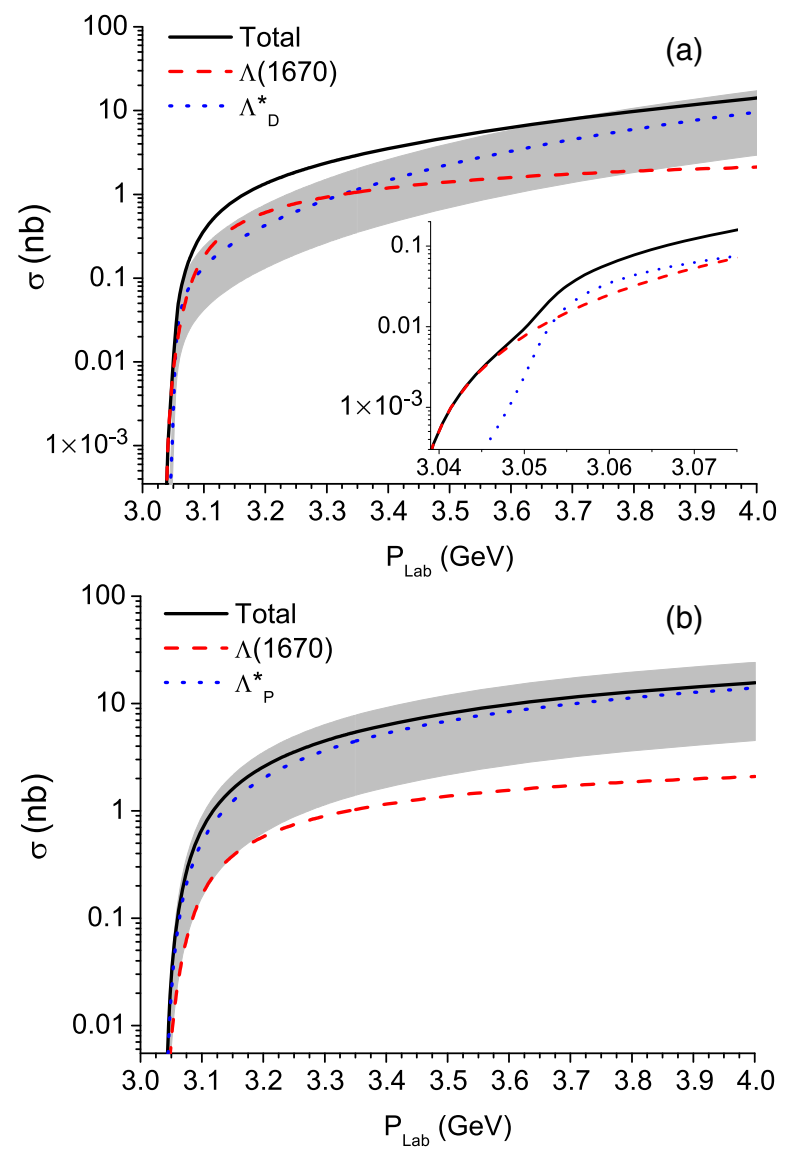

FIG. 2. Total cross sections as a function of the antiproton beam momentum obtained in Scenario I (a) and Scenario II (b). The band corresponds to the results of $\Lambda_{P / D}^{*}$ by varying the cutoff parameter for the $\Lambda_{P / D}^{*} \bar{K} N$ vertex from 0.8 to $1.4 \mathrm{GeV}$. 
energy dependence at around $P_{L a b}=3.05 \mathrm{GeV}$. As the energy increases and comes near the $\Lambda(1670) \bar{\Lambda} / \bar{\Lambda}(1670) \Lambda$ threshold, the contribution of the $\Lambda(1670)$ becomes dominant again. But at higher energies, the $\Lambda_{D}^{*}$ 's contribution exceeds the contribution of the $\Lambda(1670)$ once again. It should be noted that at the near threshold region the energy dependence due to FSI should also play an important role. Since such effects are not considered in this work, the discussions presented above only serve to show the production of $\Lambda_{D}^{*}$ may cause significant energy dependence in the total cross sections. Compared to the role of the $\Lambda_{D}^{*}$ in the $K^{-} p \rightarrow \eta \Lambda$ reaction, its role is significantly enhanced in the present reaction. The enhancement is mainly due to the D-wave nature of the $\Lambda_{D}^{*} \bar{K} N$ coupling and the large threshold momentum of the present reaction. At the near threshold region, the vertex function of the $\Lambda_{D}^{*} \bar{K} N$ vertex is roughly proportional to $p_{t h}^{2}$, where $p_{t h}$ is the threshold momentum of the reaction in the center of mass frame. Therefore, the large threshold momentum of the present reaction makes the contribution of the $\Lambda_{D}^{*}$ much more significant than that in the $K^{-} p \rightarrow \eta \Lambda$ reaction, where the threshold momentum is a factor of 4 smaller. There is no such enhancement for the s-wave state $\Lambda(1670)$, so the role of the $\Lambda_{D}^{*}$ becomes more important in the present reaction. The final results for the $\Lambda_{D}^{*}$ contribution certainly still rely on other model parameters. In our model, it may come from the cutoff parameter for the $\Lambda^{*} \bar{K} N$ vertex. To obtain the results shown in Fig. 2(a), we have adopted $\Lambda_{K}=1.1 \mathrm{GeV}$ in the $\Lambda_{D}^{*} \bar{K} N$ vertex as that for the $\Lambda(1670) \bar{K} N$ vertex in the calculations. To check the dependence on this parameter, we have also allowed the cutoff parameter for the $\Lambda_{D}^{*} \bar{K} N$ vertex varying from 0.8 to $1.4 \mathrm{GeV}$, which results in the band shown in the figure. In Scenario II, our results [Fig. 2(b)] show that the production of the $\Lambda_{P}^{*}$ dominates this reaction even at the near threshold region. In fact, in our fitting of the $K^{-} p \rightarrow \eta \Lambda$ reaction data, we also find, even though the $\Lambda(1670)$ gives the dominant contribution, the $\Lambda_{P}^{*}$ contribution is significant as well. Compared to the s-wave state $\Lambda(1670)$, the contribution of the $\Lambda_{P}^{*}$ in the present reaction is also enhanced due to the large threshold momentum as mentioned above. The band in the Fig. 2(b) shows the uncertainties due to the cutoff parameter for the $\Lambda_{P}^{*} \bar{K} N$ vertex by varying it from 0.8 to $1.4 \mathrm{GeV}$.

In Fig. 3, we show the differential cross sections obtained in Scenario I at $P_{l a b}=3.84 \mathrm{GeV}$, where the FSI of $\Lambda \bar{\Lambda}$ is expected to be small. As can be seen from the figure, there is a sharp peak appearing in the $M_{\Lambda \eta}$ spectrum. Compared to the small bump shown in the total cross sections of the $K^{-} p \rightarrow \eta \Lambda$ reaction (see Fig. 2 of Ref. [3]), the signal of the $\Lambda_{D}^{*}$ is significantly enhanced here as expected from the total cross sections shown in Fig. 2(a). The angular distribution of $\eta$ is studied in the $\Lambda \eta$ rest frame and the $\theta_{C M}$ is defined as the angle of the $\eta$ momentum relative to the beam direction. The angular distribution shows an asymmetry at forward and backward angles. The forward
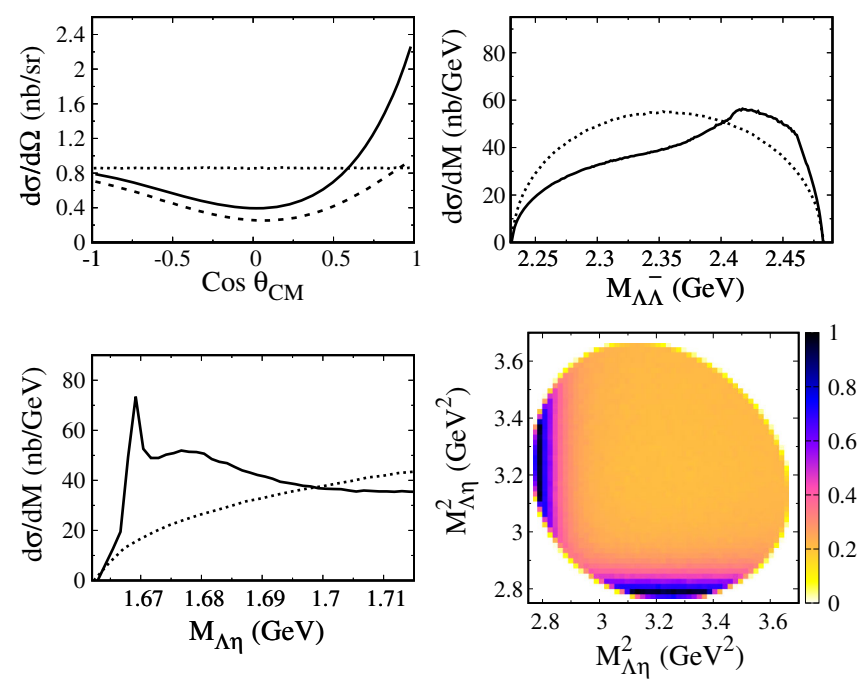

FIG. 3. Predictions for the angular distribution of $\eta$ in the $\Lambda \eta$ rest frame, the spectrum of $M_{\Lambda \bar{\Lambda}}$, the spectrum of $M_{\Lambda \eta}$ and Dalitz plot for Scenario I at $P_{\text {lab }}=3.84 \mathrm{GeV}$. The predicted results are shown by the solid lines and compared with the phase space distribution (dotted lines). The dashed line of the $\eta$ angular distribution represents the results with imposing a cut $M_{\bar{\Lambda} \eta}>1.75 \mathrm{GeV}$ on the invariant mass of $\bar{\Lambda} \eta$.

peak is mainly caused by the $\eta$ meson originated from the decay of the $\bar{\Lambda}$ resonances. While, the backward enhancement is caused by the $\Lambda_{D}^{*}$ and its interference with other contributions. If we eliminate the contribution from the $\bar{\Lambda}$ resonances by a cut with requiring $M_{\bar{\Lambda} \eta}>1.75 \mathrm{GeV}$, the asymmetry can be significantly reduced. The remaining concave-up shape of the angular distribution indicates the higher partial wave contributions from the $\Lambda_{D}^{*}$ (see also Fig. 5 for comparison). We have also checked even if we adopt $\Lambda_{K}=0.8 \mathrm{GeV}$ in the calculations, there is still a clear bump relative to the enhancement caused by the $\Lambda(1670)$ in the $M_{\Lambda \eta}$ spectrum.

The corresponding results for Scenario II are presented in Fig. 4. In this case, there is no clear peak of the $\Lambda_{P}^{*}$ in the $M_{\Lambda \eta}$ spectrum. This is mainly because the $\Lambda_{P}^{*}$ in our model lies very close to the $\Lambda \eta$ threshold and has a relatively large width $(\sim 11 \mathrm{MeV})$. The enhancement in the $M_{\Lambda \eta}$ spectrum compared to the phase space distribution is caused by a coherent sum of the contributions of the $\Lambda(1670)$ and the $\Lambda_{P}^{*}$. On the other hand, the angular distribution of $\eta$ shows distinct features compared to the results without the $\Lambda_{P}^{*}$ contribution (Fig. 5). After eliminating the $\bar{\Lambda}^{*}$ contribution as done for Scenario I, the structure shown in the $\eta$ angular distribution in the $\Lambda \eta$ rest frame also clearly indicates the higher partial wave contribution from the $\Lambda_{P}^{*}$. Compared to the results shown in Fig. 3, we find that the $\eta$ angular distributions have similar patterns in these two cases. Thus it is difficult to identify the quantum numbers of the narrow resonance by only analyzing the angular distributions, and the polarization data may be needed. A detailed study on 

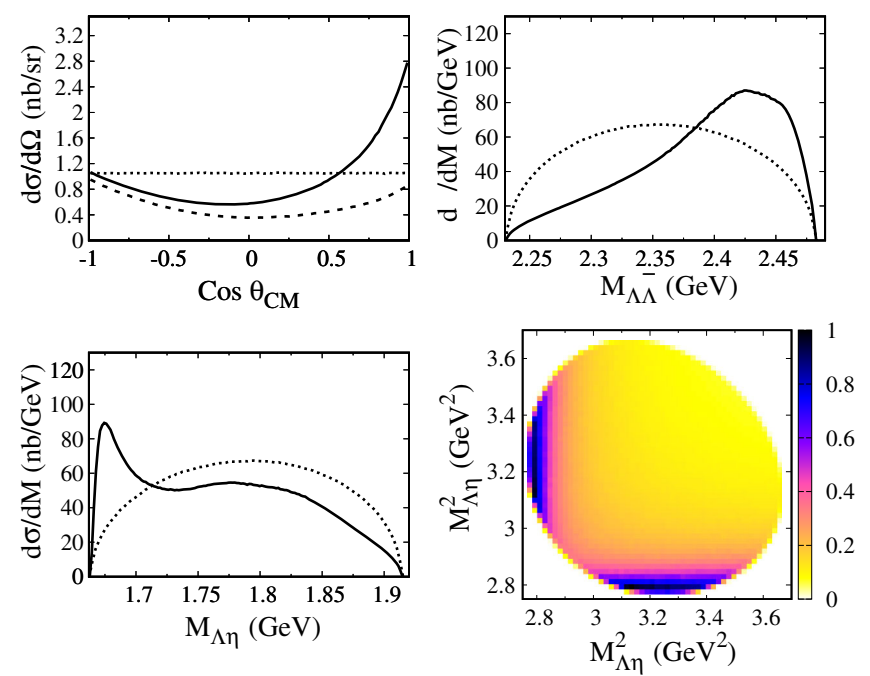

FIG. 4. Same as Fig. 3 but for Scenario II.

the polarization observables will rely on a more rigorous treatment of both ISI and FSI and is out of the scope of present work. However, as can be seen from the figures an accurate measurement of the Dalitz plot or invariant mass spectrums can still offer valuable information about the narrow resonance, since the Scenarios I and II predict distinct features in these observables.

Finally, in Fig. 5 we show the results with only considering the $\Lambda(1670)$ contribution at $P_{\text {lab }}=3.84 \mathrm{GeV}$ for comparisons. As can be seen from the figure, there is a clear enhancement caused by the $\Lambda(1670)$ appearing in the $M_{\eta \Lambda}$ spectrum. The corresponding enhancement in the Dalitz plot is also significant. After eliminating the $\bar{\Lambda}^{*}$ contributions, the angular distribution of the final $\eta$ in the $\eta \Lambda$ rest frame is roughly flat. Compared to the corresponding $\eta$ angular distributions(dashed line) in Scenarios I and II, the significant curvature shown in the $\eta$ angular distribution in the rest frame of the $\eta \Lambda$ system can be looked as the evidence for the existence of the new $\Lambda$ resonance. It is also worth noting that even though the $K^{*}$ exchange contribution is included in the calculations, we find its contribution is very small and can be neglected.
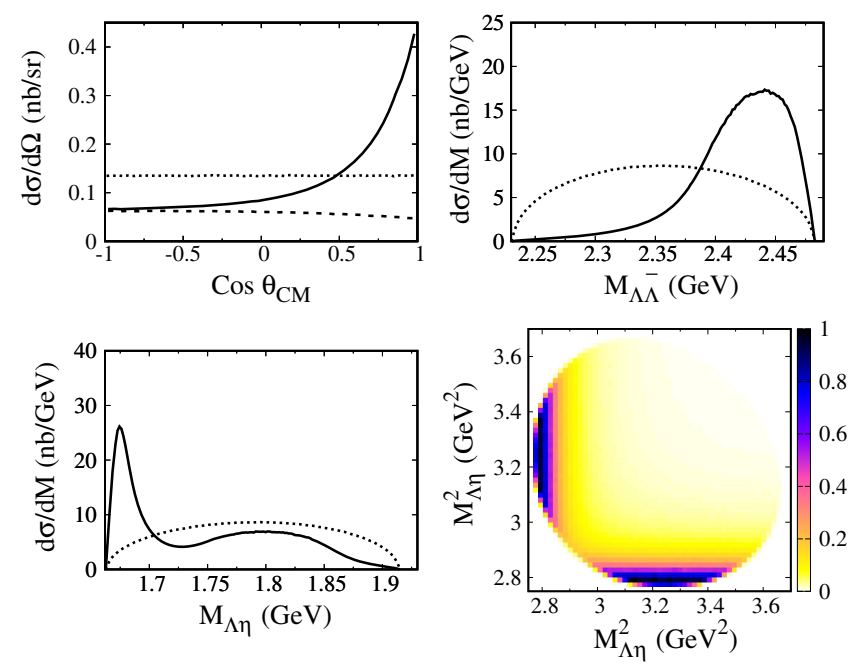

FIG. 5. Same as Fig. 3 but for Scenario III.

\section{CONCLUSION}

In this work, we investigate the production of $\Lambda / \bar{\Lambda}$ resonances in the $p \bar{p} \rightarrow \Lambda \bar{\Lambda} \eta$ reaction within an effective Lagrangian approach and isobar model. Especially, we investigate the possibility to verify the existence of a new narrow $\Lambda$ resonance found in the $K^{-} p \rightarrow \eta \Lambda$ reaction near threshold. Based on our model calculations, we find the narrow resonance, if exists, can give significant contribution in this reaction and the total cross sections of this reaction is found to be roughly at the order of $0.1-10 \mathrm{nb}$ at $P_{\text {lab }}=3.1-4 \mathrm{GeV}$. Thus the measurements of this reaction will offer a good opportunity to verify the existence of this resonance. The predictions can be tested in the future by the PANDA experiment.

\section{ACKNOWLEDGMENTS}

We acknowledge the support from the National Natural Science Foundation of China under Grants No. U1832160 and No. 11375137, the Natural Science Foundation of Shaanxi Province under Grants No. 2015JQ1003 and No. 2019JM-025, and the Fundamental Research Funds for the Central Universities.
[1] M. Tanabashi et al. (Particle Data Group), Phys. Rev. D 98, 030001 (2018).

[2] B.-C. Liu and J.-J. Xie, Phys. Rev. C 85, 038201 (2012).

[3] B.-C. Liu and J.-J. Xie, Phys. Rev. C 86, 055202 (2012).

[4] H. Kamano, S. X. Nakamura, T.-S. H. Lee, and T. Sato, Phys. Rev. C 90, 065204 (2014).

[5] H. Kamano, S. X. Nakamura, T.-S. H. Lee, and T. Sato, Phys. Rev. C 92, 025205 (2015); 95, 049903 (2017).
[6] S. B. Yang et al. (Belle Collaboration), Phys. Rev. Lett. 117, 011801 (2016).

[7] X. H. Liu, G. Li, J. J. Xie, and Q. Zhao, Phys. Rev. D 100, 054006 (2019).

[8] M. F. M. Lutz et al. (PANDA Collaboration), arXiv:0903 3905.

[9] J. He, Z. Ouyang, X. Liu, and X. Q. Li, Phys. Rev. D 84, 114010 (2011). 
[10] J. Haidenbauer and G. Krein, Phys. Lett. B 687, 314 (2010).

[11] R. Shyam and H. Lenske, Phys. Rev. D 90, 014017 (2014).

[12] Y. Y. Wang, Q. F. Lü, E. Wang, and D. M. Li, Phys. Rev. D 94, 014025 (2016).

[13] J. Haidenbauer and G. Krein, Phys. Rev. D 95, 014017 (2017).

[14] J. Z. Wang, H. Xu, J. J. Xie, and X. Liu, Phys. Rev. D 96, 094004 (2017).

[15] K. Nakayama, Y. Oh, and H. Haberzettl, J. Korean Phys. Soc. 59, 224 (2011).

[16] Y. Oh and H. Kim, Phys. Rev. C 73, 065202 (2006).

[17] Y. Oh and H. Kim, Phys. Rev. C 74, 015208 (2006).
[18] V. G. J. Stoks and T. A. Rijken, Phys. Rev. C 59, 3009 (1999).

[19] T. A. Rijken, V. G. J. Stoks, and Y. Yamamoto, Phys. Rev. C 59, 21 (1999).

[20] L. Y. Xiao, Q. F. Lü, J. J. Xie, and X. H. Zhong, Eur. Phys. J. A 51, 130 (2015).

[21] V. Baru, A. M. Gasparyan, J. Haidenbauer, C. Hanhart, A. E. Kudryavtsev, and J. Speth, Phys. Rev. C 67, 024002 (2003).

[22] C. Hanhart, Phys. Rep. 397, 155 (2004).

[23] M. L. Goldberger and K. M. Watson, Collision Theory (Wiley, New York, 1964), p. 544.

[24] W. Roberts, Z. Phys. C 49, 633 (1991).

[25] N. J. Sopkovich, Nuovo Cimento 26, 186 (1962). 